\title{
Massless black hole pairs in string theory
}

\author{
R. Emparan \\ Department of Physics \\ University of California \\ Santa Barbara, CA 93106 \\ emparan@cosmic1.physics.ucsb.edu
}

\begin{abstract}
We analyze the zero mass black holes that arise as classical solutions to low energy heterotic string theory. Though these solutions contain naked singularities, it has been conjectured that they should be allowed in the theory. We find a solution describing a pair of oppositely charged massless black holes in uniformly accelerated motion under no external force. By analytically continuing the solution to Euclidean time, we find an instanton mediating the pair production of these objects in Minkowski space. We analyze the creation rate, and discuss some consequences of the result.
\end{abstract}

UCSBTH-96-17

hep-th/9607102 
Among the many solitonic excitations that have been recently shown to play a crucial role in the non-perturbative aspects of string theory, some of the most remarkable ones are zero mass extreme black holes. They first attracted a great deal of attention when Strominger argued that they were needed in order to understand the conifold singularities that appear in the low energy Lagrangian describing the moduli space of Calabi-Yau vacua of (type II) string theory [1]. The origin of these singularities can be explained as coming from the integration of quantum loops of black holes that become massless precisely at the conifold point. One is then led to include the extremal black holes in the theory in the same footing as elementary particles.

On the other hand, several classical pointlike solutions of heterotic string theory have been recently found which have vanishing ADM mass, as measured at infinity, but nevertheless carry electric and magnetic charges, and follow timelike, instead of lightlike, trajectories [2, 3, 4, 5]. These solutions are extremal and saturate Bogomolnyi bounds. One could be tempted to relate them to the massless black holes described above. However, we should keep in mind that Strominger's massless holes arise only when the low energy action becomes singular and the semiclassical description breaks down, whereas the classical massless holes are solutions of a well-defined, non-singular theory. In the following, we will focus exclusively on the latter, classical massless holes.

A problem posed by these solutions is the presence of naked singularities at the position of the hole. These are certainly required to account for the masslessness of these objects, and are related also to other striking features. These putative massless black holes are peculiarly distinct from the more ordinary massive black holes. Their gravitational mass is zero only when measured at infinity. At finite distances, they exert a repulsive force on massive test particles [3], and in this sense they hardly deserve to be described as 'black'. Another puzzling feature of these massless black holes concerns their kinematical properties. The string theory massless hole solutions found thus far describe them at rest. It is not clear how this state can be changed; for example, Newton's law does not provide an answer to whether a zero mass hole accelerates when an external force field is applied to it. How can such objects be created? They do not seem to be the extremal limit of any nonextreme black hole. Therefore, it is unlikely that they can appear as the last stage in the evaporation of a larger, non-extremal black hole. Due to their masslessness and repulsive interactions, it is also difficult to imagine how they 
can be formed in gravitational collapse. There remains the possibility that they are created in pairs. This mechanism could be analyzed by applying the Euclidean instanton techniques used in recent years to study black hole pair creation.

In the present work we address two of these issues, namely, the accelerated motion of the massless holes, and their spontaneous pair production. The approach to both problems will be based on a solution describing a pair of massless black holes following uniformly accelerated (timelike) trajectories. A striking property of the solution is that no external force is needed to accelerate the holes. The Lorentzian solution describing this accelerated motion can be analytically continued to Euclidean time and then used to construct an instanton that describes the decay of the Minkowski vacuum by spontaneous formation of pairs of massless black holes ${ }^{1}$.

We find it convenient to present the massless hole solutions from the same point of view as in [5], i.e., as bound states of positive and negative mass objects. The starting point in this interpretation is a theory with four $U(1)$ gauge fields and three scalars, described by the action [8]

$$
\begin{aligned}
I & =\frac{1}{16 \pi G} \int d^{4} x \sqrt{-g}\left\{R-\frac{1}{2}\left[(\partial \eta)^{2}+(\partial \sigma)^{2}+(\partial \rho)^{2}\right]\right. \\
& \left.-\frac{e^{-\eta}}{4}\left[e^{-\sigma-\rho} F_{1}^{2}+e^{-\sigma+\rho} F_{2}^{2}+e^{\sigma+\rho} F_{3}^{2}+e^{\sigma-\rho} F_{4}^{2}\right]\right\},
\end{aligned}
$$

which arises as a truncation of the low energy effective action of heterotic string theory compactified on a six-torus. The equations of motion of this theory are solved by

$$
\begin{aligned}
d s^{2} & =-\left(\Delta_{1} \Delta_{2} \Delta_{3} \Delta_{4}\right)^{-1} d t^{2}+\left(\Delta_{1} \Delta_{2} \Delta_{3} \Delta_{4}\right) d \mathbf{x}^{2}, \\
e^{-\eta} & =\frac{\Delta_{1} \Delta_{3}}{\Delta_{2} \Delta_{4}}, e^{-\sigma}=\frac{\Delta_{1} \Delta_{4}}{\Delta_{2} \Delta_{3}}, e^{-\rho}=\frac{\Delta_{1} \Delta_{2}}{\Delta_{3} \Delta_{4}}, \\
F_{1 / 3 t j} & =\partial_{j} \Delta_{1 / 3}^{-2}, \quad \tilde{F}_{2 / 4 t j}=\partial_{j} \Delta_{2 / 4}^{-2},
\end{aligned}
$$

\footnotetext{
${ }^{1}$ Massless black holes in uniform acceleration have been also considered in [6] in the context of theories with imaginary electric charge. However, these massless black holes are not extremal, and no instanton describing their pair creation could be constructed. It has also been argued in [7] that pair creation of (Kaluza-Klein) massless monopoles can take place in a similar context, namely, in theories with internal time directions. In these papers the massless objects are more intended to show the pathological features of the theories in which they appear.
} 


$$
\Delta_{i}=\left(1+\frac{q_{i}}{r}\right)^{\frac{1}{2}} \quad i=1, \ldots, 4
$$

where $\tilde{F}_{2 / 4}=e^{-\eta \pm(-\sigma+\rho)} * F_{2 / 4}, * F$ being the Hodge dual of $F$. This notable solution, first found in [9] (see also [10]) and later in [8], suggests that black hole configurations can be thought of as composites of four basic building blocks, each charged under a different Maxwell field [8]. In particular, the black hole solutions of single scalar-Maxwell theories with $a=\sqrt{3}, 1,1 / \sqrt{3}, 0$ can be obtained as bound states of one, two, three or four Kaluza-Klein black holes. Notice that the constituents do not exert any net force on each other and therefore can be freely superimposed. The ADM mass of these configurations is

$$
m=\frac{1}{4} \sum_{i=1}^{4} q_{i}
$$

Therefore, it was proposed in [5] that massless solutions could be obtained by allowing for negative values of the parameters $q_{i}$. For example, by choosing $q_{1}=-q_{3}=q, q_{2}=q_{4}=0$, we obtain a bound state of negative mass/positive mass holes with zero total mass. One should keep in mind that, as stressed in [5], there is no rigorous way to define the mass of the constituents, even more in this case since negative mass objects are not expected to exist in isolation.

We have found that, remarkably, the bound state interpretation can also be extended to a more complicated class of geometries, the C-type metrics describing accelerating black holes. The solution is

$$
\begin{aligned}
d s^{2} & =\frac{1}{A^{2}(x-y)^{2}}\left[\mathcal{F}(x)\left(\frac{1-y^{2}}{\mathcal{F}(y)} d t^{2}-\frac{\mathcal{F}(y)}{1-y^{2}} d y^{2}\right)\right. \\
& \left.+\mathcal{F}(y)\left(\frac{\mathcal{F}(x)}{1-x^{2}} d x^{2}-\frac{1-x^{2}}{\mathcal{F}(x)} d \varphi^{2}\right)\right], \\
\mathcal{F}(\xi) & =\prod_{i=1}^{4} f_{i}(\xi), \quad f_{i}(\xi)=\left(1-q_{i} A \xi\right)^{\frac{1}{2}}, \\
e^{-\eta} & =\frac{f_{1}(y) f_{3}(y)}{f_{2}(y) f_{4}(y)} \frac{f_{2}(x) f_{4}(x)}{f_{1}(x) f_{3}(x)}, e^{-\sigma}=\frac{f_{1}(y) f_{4}(y)}{f_{2}(y) f_{3}(y)} \frac{f_{2}(x) f_{3}(x)}{f_{1}(x) f_{4}(x)}, \\
e^{-\rho} & =\frac{f_{1}(y) f_{2}(y)}{f_{3}(y) f_{4}(y)} \frac{f_{3}(x) f_{4}(x)}{f_{1}(x) f_{2}(x)},
\end{aligned}
$$




$$
\begin{aligned}
& F_{1 / 3 t y}=\frac{q_{1 / 3} \sqrt{1-q_{1 / 3}^{2} A^{2}}}{f_{1 / 3}(y)^{4}}, \\
& \tilde{F}_{2 / 4 t y}=\frac{q_{2 / 4} \sqrt{1-q_{2 / 4}^{2} A^{2}}}{f_{2 / 4}(y)^{4}} .
\end{aligned}
$$

This solution contains as particular cases the dilaton and $U(1)^{2}$ extremal C-metrics found in [11, 12 for $a=\sqrt{3}, 1,1 / \sqrt{3}, 0$ (some rewriting of the parameters and coordinates may be needed to bring the solutions in this form). One can also add background Melvin fields corresponding to each of the four $U(1)$ gauge fields, which also take the same factorized form, and thus obtain Ernst-type metrics. A detailed analysis of the massive solutions in background fields will be given elsewhere. For the moment we will only focus on the solution (田) with the purpose of studying the massless case.

The interpretation of the solution (4) in terms of two objects accelerating apart can be found as usual [13], so we shall be brief. We restrict the parameters to satisfy $\left|q_{i} A\right|<1 \forall i=1, \ldots, 4$. If $\left|q_{i} A\right|>1 \forall i=1, \ldots, 4$, then we find an isometry, up to a global sign, to the previous case by taking $y \rightarrow y^{-1}, x \rightarrow x^{-1}, q_{i} A \rightarrow\left(q_{i} A\right)^{-1}$. The static solution (2) can be recovered by setting $y \rightarrow-1 /(r A), t \rightarrow A t$, and then taking the limit $A \rightarrow 0$. This allows us to say that the metric contains objects in one-to-one correspondence with the solutions of (2). On the other hand, sending $q_{i} \rightarrow 0$ yields Rindler (flat) space in non-standard coordinates. If there are negative roots $y=\left(q_{i} A\right)^{-1}<-1$, they correspond to the singularities at the origin of negative mass constituents, whereas $y=-\infty$ is interpreted as the position of the horizon of a positive mass constituent. Also, $y=-1$ is a Rindler horizon. The variables are restricted to $-1 \leq x \leq 1, y_{0}<y<x$, where $y_{o}=\max _{i}\left\{-q_{i} A, 0\right\}$. Asymptotic infinity is at $x=y$; in particular, $x=y=-1$ is spacelike infinity. The parameter $A$ roughly measures the acceleration of the black holes, but also their separation when they become closest, which is given by (twice) $1 / A$. This solution asymptotes to flat space (with, possibly, a conical singularity, see below).

The angular part $(x, \varphi)$ of the metric, with $\varphi$ identified with period $\Delta \varphi$, has the topology of a two-sphere. In general, one can not choose $\Delta \varphi$ so as to eliminate conical singularities at both poles $x= \pm 1$. These conical singularities reflect the need of forces to accelerate the black holes, and are usually cancelled by, e.g., adding background fields. However, let us examine this 
issue closer for the metric (41), which does not contain such fields. Regularity at both poles can only be achieved if

$$
\Delta \varphi=2 \pi \mathcal{F}(1)=2 \pi \mathcal{F}(-1) .
$$

For arbitrary $A$ the equation $\mathcal{F}(1)=\mathcal{F}(-1)$ requires

$$
q_{1}+q_{2}+q_{3}+q_{4}=0
$$

and

$$
q_{1} q_{2} q_{3}+q_{1} q_{3} q_{4}+q_{2} q_{3} q_{4}+q_{1} q_{2} q_{4}=0 .
$$

But (6) is just the zero mass condition for the black holes! Though the mass of the black hole is not well defined in the non-spherical metric (雨), it should be clear that the object that is accelerating is in direct correspondence to the static, massless hole. Therefore, by choosing the parameters to satisfy these equations, we succeed in finding a solution describing two massless black holes moving with uniform acceleration in an asymptotically Minkowskian space. No external force is acting upon the black holes.

The meaning of (7) is less clear, but it is probably required to cancel the forces that appear between black holes when they are in motion. In any case, it is satisfied by the simplest and most interesting massless holes.

The parameters $q_{i}$ are not exactly equal to the physical charges of the black hole. Rather, they approximate them in the limit $A \rightarrow 0$. The physical charges $\hat{q}_{i}$ are found by integrating the (dual) field strengths on a sphere surrounding the black hole. One finds then

$$
\hat{q}_{i}=q_{i} \frac{\mathcal{F}(1)}{\sqrt{1-q_{i}^{2} A^{2}}} .
$$

An aspect worth mentioning is that the metric (4) does not become spherical as the position of the hole is approached. The hole is slightly elongated along the axis of acceleration. A similar effect was also found in [12]. This is in contrast to [11], where the geometry close to an extremal dilaton black hole in accelerated motion was found to approach exactly that of the static hole.

The bound state interpretation suggests that a small perturbation of the static massless holes could set them in runaway motion, in which the negative mass constituent chases the positive mass one (this is related to the 
old problem of "Bondi dipoles" in General Relativity, see e.g. [14]). Our Cmetric precisely describes such motion (though we should keep in mind that the solution actually describes a pair of massless holes). The elongation of the massless hole can presumably be seen as the separation between the distributions of negative and positive massess needed to maintain the continued accelerated motion of the bound state.

It might seem puzzling that the interaction between the two massless holes, which one would expect to be attractive since they form a particleantiparticle pair, does not prevent the pair from separating away. The holes are accelerated and distorted, and the forces between them are rather complicated. It is clear that these forces should be more and more negligible as we take $A \rightarrow 0$ (and therefore $1 / A \rightarrow \infty$ ). The fact that, in the absence of external forces, the holes accelerate apart instead of collapsing and annihilating each other, would suggest that their net effective interaction could in fact be repulsive 9 . Notice, however, that it is not possible to readily resort to the repulsive forces found in [3], since the massless holes cannot be treated as test particles. In any case, the solution we have found certainly incorporates all these interactions, and the absence of external forces appears neatly in the final result.

Now, the solution (雨) can be continued to Euclidean time by taking $\tau=i t$. Regularity at the acceleration horizon at $y=-1$ is achieved by taking the period of $\tau$ to be

$$
\beta=2 \pi \mathcal{F}(-1) .
$$

This Euclidean metric describes a massless black hole loop. By slicing in half the instanton, and glueing it to the time-symmetric section of the Lorentzian solution, we obtain a description of the spontaneous creation of a pair of massless black holes out of the Minkowski vacuum.

What is the creation rate for this process? This is usually taken to be given, to leading order, by $e^{-I}$, where $I$ is the classical action of the Euclidean instanton. Following [15], we decompose the action in the following terms:

$$
I=\beta H-\frac{1}{4}\left(A_{b h}+\Delta A\right)
$$

with $H$ the Hamiltonian, $A_{b h}$ the area of the black hole horizon, and $\Delta A$ the

\footnotetext{
${ }^{2}$ This might be related to the appearance of negative Euclidean action that we find below.
} 
difference in the area of acceleration horizons. The action is calculated with reference to a background which in the present case will be flat space.

The area term $A_{b h} / 4$ is associated, as usual, to the entropy of the black hole, and it has been argued that this term must be present, in string theory, for non-extreme as well as for extreme black holes [16]. A potentially troublesome issue in the case at hand is the curvature singularity at the position of the hole. There is no horizon area at that point. However, we are considering the possibility that massless hole singularities could be allowed in the theory. One could, perhaps, expect the geometry near the horizon to be modified where the curvature reaches the string scale and string effects can be expected to come into play. It has been argued that, for zero area black holes in string theory, the entropy of the black hole can be found by drawing a surface at that radius [17]. We would then find a term $S_{b h}$ enhancing the pair creation rate. Far from the horizon, string theory corrections should in any case be negligible, so that $H$ and $\Delta A$ can be computed in the classical, low energy geometry.

The Hamiltonian in (10) consists of two terms, a bulk term that vanishes on exact solutions, and a surface term. Since the spacetime is non-compact, we must introduce a boundary near infinity; care must be taken so that the boundaries of the C-metric and the flat space background are identical. When this is done, a detailed but straightforward calculation shows that the surface term in the Hamiltonian vanishes, just as in all black hole pair creation processes analyzed thus far.

We are therefore left with

$$
I=-\frac{1}{4} \Delta A-S_{b h}
$$

$\Delta A$ should give the dominant contribution to the pair creation rate. In fact, it usually gives the total contribution to the pair creation rate of extreme black holes [15]. Again, to calculate it we must carefully perform the subtraction of the areas. By introducing a boundary in the C-metric at $y=-1, x=-1+\epsilon_{c}$ and evaluating the quantities only up to second non-trivial order in $\epsilon_{c}$, the length of this boundary is

$$
l_{c}=\int d \varphi \sqrt{g_{\varphi \varphi}}=2 \pi \rho_{c}\left(1-\frac{\sum_{i} \hat{q}_{i}^{2}}{2 \rho_{c}^{2}}-\frac{\mathcal{F}(1)^{2}}{2 A^{2} \rho_{c}^{2}}\right),
$$

where the no-force conditions (6,7) have been imposed and we have defined 
$\rho_{c} \equiv \mathcal{F}(-1) \sqrt{\frac{2}{A^{2} \epsilon_{c}}}$. The boundary in the Minkowski background, written in cylindrical coordinates $(t, z, \rho, \varphi)$, is at $\rho=\rho_{M}$, and its length is $l_{M}=2 \pi \rho_{M}$. We match the boundaries in the C-metric and in Minkowski spacetime by requiring $l_{M}=l_{c}$.

The area of the acceleration horizon in the $\mathrm{C}$-metric is

$$
A_{c}=\left.\int d x d \varphi \sqrt{g_{x x} g_{\varphi \varphi}}\right|_{y=-1}=-\frac{\pi \mathcal{F}(1)^{2}}{A^{2}}+\pi \rho_{c}^{2} .
$$

In the background, the acceleration horizon is at $z=0$, and the area inside a circle $\rho=\rho_{M}$ is $A_{M}=\pi \rho_{M}^{2}$. Then we end up with the exact result

$$
\Delta A=A_{c}-A_{M}=\pi \sum_{i=1}^{4} \hat{q}_{i}^{2} .
$$

This is a striking result, and not only because of its simplicity. Notice that $\Delta A>0$, i.e., the area of the acceleration horizon in the $\mathrm{C}$-metric is bigger than in the reference metric. This is just the opposite of what happens in all the black hole creation processes studied thus far, where, in fact, this term yields a negative result and thus the suppression factor for the pair creation rate. For the massless holes, however, the leading term in the pair creation rate should be $\exp \left(\frac{\pi}{4} \sum_{i=1}^{4} \hat{q}_{i}^{2}\right)$, (times, possibly, a factor $\left.e^{S_{b h}}\right)$. Apparently, pair production of massless black holes is not suppressed, but, on the contrary, enhanced! fo. This is certainly disturbing, since it would seem to imply that if these massless holes were actually possible, the Minkowski vacuum should be highly unstable against their production in pairs.

A minor subtlety arises in that, when continuing to Euclidean time, the electric-type charges $q_{1}, q_{3}$, should also be continued to imaginary values. It has been argued, however, that in these cases a charge projection on electric states must be introduced that restores the expected electric-magnetic duality of decay rates [18]. Therefore, we still expect the Euclidean exponential pair production factor to be given by the same positive definite function (114) $)^{\text {. }}$.

\footnotetext{
${ }^{3}$ The reader should be cautioned, though, that there is no agreement between different authors as to whether the action contribution should be weighed as $e^{-I}$ or rather as $e^{-|I|}$.

${ }^{4}$ Notice that it is always possible to have a massless hole constructed out of only magnetic-type constituents, i.e., $q_{2}=-q_{4}, q_{1}=q_{3}=0$.
} 
The result is also peculiar in other respects. Normally, for extreme massive black holes, the term $-\Delta A / 4$ when expressed in terms of physical parameters reproduces the usual exponent of the Schwinger pair production rate

$$
I=\frac{\pi m^{2}}{F}(1+O(F))=\frac{\pi m}{A}(1+O(m)),
$$

where $F$ is the driving force, $F \approx m A$. In the limit of zero mass, one would expect to find vanishing action. Certainly, this is very different from the behavior that we have found. Moreover, creation of objects in slowly accelerated motion is usually strongly suppressed since the action grows larger. In contrast, the corresponding term (14) goes smoothly to a finite value as $A \rightarrow 0$. Thus, within the range $\left|q_{i} A\right|<1$, the massless black holes can be created with arbitrary acceleration. Recalling that $1 / A$ measures the separation between black holes at the moment of their production, we see that the massless holes can be created arbitrarily far from each other and we still find a finite, bounded action.

Let us now discuss some potential problems of the result we have found. The static extremal solutions are protected from quantum renormalization effects by supersymmetry. The solution (4) does not presumably preserve any supersymmetry. However, since we can create the black holes far apart from each other, they can approximate more and more the static solutions. Recent experience suggests that, in some cases, corrections in near BPS states can be kept under control [19]. We also want to remark that our analysis does not depend on the bound state interpretation. One could simply take the solution and not bother about hypothetic constituents, though the form of the solutions is certainly compelling.

This troublesome unsuppressed pair creation out of the vacuum does not readily apply to the quantum massless black holes of [1]. The fact that the asymptotically Euclidean instanton above has negative action is clearly due to the naked singularities of the massless holes. This may cast some doubt on whether these classical singular solutions shoud be allowed in the theory. As already mentioned, string effects in the region near the hole can modify the geometry and correct the total action, but it is not clear how this could change qualitatively the result. The pair production mechanism described is based on commonly accepted semiclassical instanton methods. Should one reject their validity when massless holes are present? For example, it could be objected that, usually, semiclassical instanton techniques require 
the action to be large (and positive), which is not the present case. But this is more an objection to the quantitative result than to the qualitative instability suggested by the solution above. We also remark that the unusual result $\Delta A>0$ is already present in the Lorentzian regime. With all these caveats in mind, the conclusion is that the pair creation process described clearly illustrates that allowing for classical massless solutions in the theory may be a source of instabilities.

We have benefited from helpful comments from G. Horowitz, A. Strominger, and T. Ortín. This work has been partially supported by a FPI postdoctoral fellowship from MEC (Spain), and by CICYT AEN-93-1435 and UPV 063.310-EB225/95.

\section{References}

[1] A. Strominger, Massless black holes and conifolds in string theory, Nucl. Phys. B451 (1995) 96.

[2] K. Behrndt, About a class of exact string backgrounds, Nucl. Phys. B455 (1995) 188.

[3] R. Kallosh and A. Linde, Exact supersymmetric massive and massless white holes, Phys. Rev. D52 (1995) 7137.

[4] M. Cvetic and D. Youm, Singular BPS saturated states and enhanced symmetries of four-dimensional $N=4$ supersymmetric string vacua, Phys. Lett. B359 (1995) 87.

[5] T. Ortín, Massless string theory black holes as black diholes and quadruholes, Phys. Rev. Lett. 76 (1996) 3890.

[6] G.W. Gibbons and D.A. Rasheed, Dyson-pairs and zero-mass black holes, hep-th/9604177.

[7] A. Chamblin and R. Emparan, Bubbles in Kaluza-Klein theories with space- or time-like internal directions, preprint UCSBTH-96-20, ITP96-66 and hep-th/9607236.

[8] J. Rahmfeld, Extremal black holes as bound states, Phys. Lett. B372 (1996) 198. 
[9] M. Cvetic and D. Youm, Dyonic BPS saturated black holes of heterotic string on a six-torus, Phys. Rev. D53 (1996) 584.

[10] M. Cvetic and A. Tseytlin, Solitonic strings and BPS saturated dyonic black holes, Phys. Rev. D53 (1996) 5619.

[11] F. Dowker, J.P. Gauntlett, D. Kastor, J. Traschen, Pair creation of dilaton black holes, Phys. Rev. D49 (1994) 2909; F. Dowker, J.P. Gauntlett, S.B. Giddings, and G.T. Horowitz, Pair creation of extremal black holes and Kaluza-Klein monopoles, ibid. D50 (1994) 2662.

[12] S.F. Ross, Pair production of black holes in a $U(1) \otimes U(1)$ theory, Phys. Rev. D49 (1994) 6599; Pair creation rate for $U(1)^{2}$ black holes, ibid. D52 (1995) 7089.

[13] W. Kinnersley and M. Walker, Uniformly accelerating charged mass in General Relativity, Phys. Rev. D2 (1970) 1359.

[14] W. Israel and K.A. Khan, Collinear particles and Bondi dipoles in General Relativity, Nuovo Cim. 33 (1964) 331.

[15] S.W. Hawking, G.T. Horowitz, and S.F. Ross, Entropy, area, and black hole pairs, Phys. Rev. D51 (1995) 4302.

[16] G.T. Horowitz, The origin of black hole entropy in string theory, in Proceedings of the Pacific Conference on Gravitation and Cosmology, Seoul, Korea (1996).

[17] A. Sen, Extremal black holes and elementary string states, Mod. Phys. Lett. A10 (1995) 2081.

[18] S.W. Hawking and S.F. Ross, Duality between electric and magnetic black holes, Phys. Rev. D52 (1995) 5865.

[19] G.T. Horowitz and A. Strominger, Counting states of near extremal black holes, hep-th/9602051. 